\title{
Knockdown of FOXK1 alone or in combination with apoptosis- inducing 5-FU inhibits cell growth in colorectal cancer
}

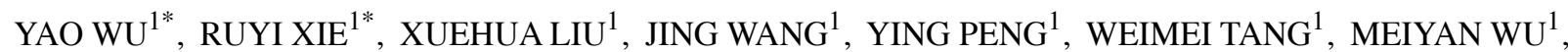 \\ PEI ZHANG ${ }^{1}$, YANG BA ${ }^{1}$, JINJUN ZHAO ${ }^{2}$, AIMIN LI ${ }^{1}$, QINGZHEN NAN ${ }^{1}$, \\ YE CHEN $^{1}$, SIDE LIU $^{1}$ and JIDE WANG ${ }^{1}$ \\ ${ }^{1}$ Guangdong Provincial Key Laboratory of Gastroenterology, Department of Gastroenterology, Nanfang Hospital, \\ Southern Medical University; ${ }^{2}$ Department of Rheumatism, Nanfang Hospital, \\ Southern Medical University, Guangzhou, Guangdong 510515, P.R. China
}

Received March 22, 2016; Accepted May 7, 2016

DOI: 10.3892/or.2016.5041

\begin{abstract}
Forkhead box K1 (FOXK1) is a member of the FOX transcription factor family, which plays an important role in oncogenesis. However, the exact function and mechanism of FOXK1 in human colorectal cancers (CRCs) remain unclear. In the present study, we first screened for potential FOXK1 target genes by ectopically expressing FOXK1 in SW480 cells and examined the subsequent changes in the expression levels of major oncogenes using RT-PCR. We also evaluated the effects of FOXK1 regulation on growth and apoptosis. In addition, we investigated the biological impact of FOXK1 knockdown on CRC cells in vitro and in vivo. We found that FOXK1 overexpression increased the expression of multiple oncogenes in vitro. FOXK1 promoted serum-dependent and anchorage-dependent and -independent cell growth. Knockdown of FOXK1 induced G0/G1 cell cycle arrest in CRC cells. Moreover, FOXK1 suppression induced apoptosis and increased cell susceptibility to 5-fluorouracil (5-FU)-induced apoptosis. Furthermore, a xenograft model was established to explore FOXK1 shRNA-mediated tumorigenesis in vivo. A strong antitumorigenic effect of FOXK1-shRNA was enhanced when combined with 5-FU treatment. These findings implicate FOXK1 as a cell cycle and growth modulator that inhibits apoptosis in colon cancer cells. FOXK1-shRNA may serve as a novel and potent therapeutic agent, alone or with 5-FU, against colon cancer.
\end{abstract}

Correspondence to: Dr Jide Wang or Dr Side Liu, Department of Gastroenterology, Nanfang Hospital, Southern Medical University, Guangzhou, Guangdong 510515, P.R. China

E-mail: jidewang55@163.com

E-mail: liuside@163.com

${ }^{*}$ Contributed equally

Key words: FOXK1, colorectal cancer, cell cycle, apoptosis, TUNEL

\section{Introduction}

As one of the major causes of cancer-related mortality worldwide, colorectal cancer (CRC) is surgically curable at early stages, but advanced disease at the metastatic stage is associated with high mortality rates $(1,2)$. CRCs progress from local adenomas in the intestinal epithelium to invasive carcinomas that typically metastasize to the liver (3). Approximately half of all patients with local CRCs may develop metastases. Current therapies show a 5-year survival of less than $5 \%$ for metastatic disease (4).

The forkhead box (Fox) gene family encodes a large and diverse group of transcription factors that share certain characteristics in a conserved, $\sim 100$ amino acid DNA-binding motif known as the forkhead or winged helix domain; over 150 proteins with forkhead domains have been identified, comprising at least 17 subclasses, to date $(5,6)$. Through the transcriptional control of gene expression, many FOX protein members reportedly play important roles in embryonic development and organogenesis and in the regulation of various physiological processes, such as the cell cycle (7), progression (8), cell survival (9), cellular metabolism (10), life span (11) and immune responses (12). Consequently, dysregulation of the function, subcellular localization and expression of FOX transcription factors leads to the development and progression of diseases, particularly cancer $(13,14)$. Several other FOX family proteins have been shown to have either a tumor-promoting [FOXA1 (15), FOXQ1 (16), FOXM1 (17) and FOXC2 (18)] or -suppressing [FOXD3 (19), FOXP1 (20), FOXO1 (21) and FOXP2 (22)] role in the cellular signaling that is associated with proliferation and/or suppression.

The human forkhead box K1 (FOXK1) gene encodes predicted proteins that are most homologous to the mouse myocyte nuclear factor MNF/Forkhead box K1 (Foxk1) protein. FOXK1 is predominantly expressed in many malignant tissues and in the brain, colon and lymph nodes (23). This gene has been implicated in normal and neoplastic developmental processes. Wang et al demonstrated that FOXK1 and FOXK2 positively regulate $\mathrm{Wnt} / \beta$-catenin signaling by translocating 
Dishevelled (DVL) into the nucleus. Moreover, FOXK1 and FOXK2 protein levels are elevated in human CRCs (24). However, the underlying mechanism of FOXK1 induction in multiple cellular events, such as growth, apoptosis and chemotherapy resistance, remains unclear.

In the present study, we demonstrated a novel and effective way to knock down FOXK1, thereby significantly inducing apoptosis, inhibiting tumorigenesis and tumor growth and strongly enhancing the antitumor activity of 5-fluorouracil (5-FU) in vitro and in vivo. Our results showed that lenti-shRNA-FOXK1 is a promising candidate for the gene therapy of colon cancer.

\section{Materials and methods}

Reagents and cell culture. 5-FU was purchased from Sigma-Aldrich (St. Louis, MO, USA). Mouse antibodies against FOXK1 (G-4), cyclin D1 (A-12), CDK4 (H-22), CDK6 (C-21), caspase 3 p11 (C-6), caspase 8 p18 (E-8), caspase 9 p35 (A-9), PARP-1 (H-300), GAPDH (G-9), horseradish peroxidase-conjugated anti-goat $\operatorname{IgG}$, and anti-mouse $\operatorname{IgG}$ were purchased from Santa Cruz Biotechnology (Santa Cruz, CA, USA). Culture reagents were purchased from Invitrogen (Carlsbad, CA, USA). The colon cancer cell lines SW480 and SW1116 were obtained from the American Type Culture Collection (ATCC; Rockville, MD, USA) and were cultured as previously described (25). The cells were maintained at $37^{\circ} \mathrm{C}$ with $5 \% \mathrm{CO}_{2}$ in a humidified incubator and were subcultured using $0.25 \%$ trypsin every 2-3 days, before confluence was reached.

Constructs and establishment of stable transfectants. Complementary DNA (cDNA) corresponding to full-length FOXK1 was obtained by RT-PCR amplification of normal human testis cDNA with primers specific to FOXK1. The PCR products were subcloned into the mammalian expression vector pcDNA3.1 (Invitrogen).

Toestablish stable cell lines, cells transfected with the empty pcDNA3.1 vector or with pcDNA3.1-FOXK1 were passaged at 1:15 (vol/vol) and were cultured in RPMI-1640 medium supplemented with geneticin (G418; Calbiochem, Darmstadt, Germany) at $1,000 \mu \mathrm{g} / \mathrm{ml}$ for 4 weeks. Stably transfected clones were selected by immunoblotting for FOXK1 expression and were maintained in medium containing $800 \mu \mathrm{g} / \mathrm{ml}$ G418 for additional studies.

RNA isolation and reverse transcription-PCR analysis. Cells were harvested, and total RNA was extracted using TRIzol reagent (Gibco-BRL and Life Technologies) (25). RNA was reverse transcribed to cDNA using the ThermoScript RT system reagent (Gibco-BRL) according to the manufacturer's instructions. PCR was performed using $2 \mu \mathrm{l}$ of the resulting cDNA and $0.3 \mathrm{U}$ of hot start DNA polymerase. Hot start PCR was performed for 32 cycles, with an initial $95^{\circ} \mathrm{C}$ denaturation for $3 \mathrm{~min}$ before cycles of $94^{\circ} \mathrm{C}$ denaturation for $45 \mathrm{sec}, 55^{\circ} \mathrm{C}$ annealing for $45 \mathrm{sec}$, and $72^{\circ} \mathrm{C}$ elongation for $45 \mathrm{sec}$ and a final extension of $10 \mathrm{~min}$.

Transient siRNA transfection. Ablation of FOXK1 was performed by transfection with small interfering RNA (siRNA) duplex oligos, which were synthesized by GenePharma
(Shanghai, China). Control siRNA (scrambled RNA) and FOXK1-specific siRNA [sense, 889-GAGACAGCCC CAAGGAUGA (dTdT)-908 and antisense, 908-UCAUCCUU GGGGCUGUCUC(dTdT)-889] were transfected using Lipofectamine 2000 (Invitrogen). Forty-eight hours after transfection, western blot analysis was performed.

Western blot analysis. For western blot analysis, cells were harvested $48 \mathrm{~h}$ after transfection and lysed in lysis buffer [10 mM Tris- $\mathrm{HCl}$ (pH 7.4), $1 \%$ SDS, $10 \%$ glycerol, $5 \mathrm{mM}$ $\mathrm{MgCl}_{2}, 1 \mathrm{mM}$ phenylmethylsulfonyl fluoride, $1 \mathrm{mM}$ sodium orthovanadate, $5 \mu \mathrm{g} / \mathrm{ml}$ leupeptin and $21 \mu \mathrm{g} / \mathrm{ml}$ aprotinin). A total of $30 \mu \mathrm{g}$ of each protein lysate was separated by SDS-PAGE and transferred onto a polyvinylidene fluoride (PVDF) membrane. The primary antibodies were diluted according to the manufacturer's recommendations. The antibodies were then visualized using an enhanced chemiluminescence detection system.

Flow cytometric analysis. For cell cycle analysis, SW480 cells were reverse transfected into 6-well dishes and were incubated for $48 \mathrm{~h}$ under standard growth conditions. Cells were fixed for $90 \mathrm{~min}$ with $70 \%(\mathrm{v} / \mathrm{v})$ pre-cooled ethanol $\left(-20^{\circ} \mathrm{C}\right)$ and harvested by centrifugation $\left(4^{\circ} \mathrm{C}, 5 \mathrm{~min}, 1,000 \mathrm{x} \mathrm{g}\right)$. Staining was performed using a $69-\mu \mathrm{M}$ propidium iodide (PI) solution in phosphate-buffered saline (PBS) containing RNase A $(0.6 \mu \mathrm{g} / \mathrm{ml})$ for $30 \mathrm{~min}$ at $37^{\circ} \mathrm{C}$. The cell cycle distribution was calculated from the resultant DNA histograms using FlowJo software.

Apoptosis was detected using the Annexin V-FITC kit (Trevigen, Inc., Gaithersburg, MD, USA) according to the manufacturer's instructions. Briefly, cells with various treatments were collected and stained with Annexin V-FITC and PI in the dark for $15 \mathrm{~min}$ before being subjected to flow cytometry and analyzed using FlowJo software.

Cell growth assay. Cells were seeded at $5.0 \times 10^{4} /$ well into 96-well plates. Cell proliferation was determined by measuring the absorption of the cell proliferation reagent WST-1 (Roche Molecular Biochemicals, Basel, Switzerland) according to the manufacturer's protocol. The ratio of the absorbance of treated cells at $450 \mathrm{~nm}$ relative to that of the untransfected cells was calculated and expressed as the proliferation index. Each treatment was performed in quadruplicate, and the proliferation values were expressed as the mean \pm SEM. The anchorageindependent cell growth assay was performed as previously described (25). Briefly, stable transfectants were seeded at $2 \times 10^{3} /$ well into 6 -well plates and cultured for 14 days. Cell colonies were microscopically visualized after staining with $0.005 \%$ crystal violet. Each treatment was conducted in triplicate, and 3 independent experiments were performed.

Morphological detection of apoptosis. Apoptotic cell death was morphologically evaluated as previously described, with some modifications. Cells were fixed for $5 \mathrm{~min}$ in 3\% paraformaldehyde in PBS. After air drying, the cells were stained for $10 \mathrm{~min}$ in Hoechst $33258(10 \mu \mathrm{g} / \mathrm{ml})$, mounted in $50 \%$ glycerol containing $20 \mathrm{mM}$ citric acid and $50 \mathrm{mM}$ orthophosphate, and stored at $-20^{\circ} \mathrm{C}$ before analysis; nuclear morphology was evaluated using a Zeiss IM 35 fluorescence microscope. 
Construction of lentiviral vectors with FOXK1 short hairpin $R N A$. To further investigate the effect of siRNA-induced downregulation of FOXK1 expression on the in vivo tumor growth of CRC, a FOXK1-RNAi lentiviral vector (GV208-FOXK1-shRNA) was constructed by Shanghai GeneChem Co., Ltd. (Shanghai, China). Double-stranded oligonucleotides encoding human FOXK1-vshRNA (NM_001037165; CCGGGAGACAGCCCCAAGGATGATC AAGAGTCATCCTTGGGGCTGTCTCTTTTTG) were annealed and inserted into the short hairpin RNA (shRNA) expression vector GV208-GFP (Shanghai GeneChem Co, Ltd.). A GFP lentiviral vector (GV208-GFP) was used as a negative control. Clone identity was verified by sequencing.

Recombinant lentiviral vector was produced by co-transfecting HEK293FT cells with the lentiviral expression vector and packing the plasmid mix using Lipofectamine ${ }^{\mathrm{TM}} 2000$ according to the manufacturer's instructions. Infectious lentiviral particles were harvested at $48 \mathrm{~h}$ post-transfection and were then filtered through $0.45 \mu \mathrm{m}$ cellulose acetate filters. The virus was concentrated, and the titer was determined by serial dilution of $293 \mathrm{~T}$ cells.

For lentivirus transduction, the SW480 cells were subcultured at $1 \times 10^{5}$ cells/well into 6 -well culture plates. Cells were transduced with the FOXK1-siRNA-expressing (FOXK1siRNA) or scramble (scr)-siRNA-expressing lentivirus at a multiplicity of infection (MOI) of 50. Cells were then harvested $72 \mathrm{~h}$ after infection, and the transduction efficiency was evaluated by counting the percentage of GFP-positive cells.

In vivo tumor growth assay. The present study was conducted in strict accordance with the recommendations in the Guide for the Institutional Animal Care and Use Committee (IACUC), and the protocol was approved by the Committee on the Ethics of Animal Experiments of Nanfang Hospital. All of the surgeries were performed under sodium pentobarbital anesthesia, and all efforts were made to minimize suffering. After the surgery, all of the nude mice were euthanized by sodium pentobarbital anesthesia.

SW480 CRC cells were transduced in vitro at an MOI of 50 with lenti-scr-shRNA or lenti-FOXK1-shRNA infected with NS. Twelve hours after transduction, $5 \times 10^{6}$ viable cells expressing lenti-scr-shRNA or lenti-FOXK1-shRNA were injected into the right flanks of 6 -week-old female nude mice, respectively. On day 28 after inoculation, the mice were sacrificed, and the tumors were dissected and weighed. Immunohistochemical analysis was performed using antiKi-67 and anti-CD105 antibodies.

For the other 3 groups of mice, lenti-scr-shRNA, lenti-scrshRNA + 5-FU or lenti-FOXK1 + 5-FU were directly injected into the tumors at 3 different locations using a 10-ml micro-syringe (Hamilton, Reno, NV, USA). Three mice (3 injections) were included in each group. The volume of each tumor was calculated as follows: $\mathrm{V}=(1 / 2) \mathrm{R} 1^{2} \mathrm{R} 2$, where $\mathrm{R} 1$ is radius $1, \mathrm{R} 2$ is radius 2 , and $\mathrm{R} 1<\mathrm{R} 2$. The mice were sacrificed, and the tumors were dissected and weighed on day 28 after inoculation. The tumors were then removed, fixed in formalin, embedded in paraffin and subjected to terminal deoxynucleotidyltransferase-mediated deoxyuridine triphosphate-digoxigenin nick-end labeling (TUNEL).
In situ detection of apoptotic cells by the TUNEL assay. Cell apoptosis in tumor xenograft tissues was detected by TUNEL staining. Tumor tissues from xenografts were excised and immediately formalin-fixed. TUNEL staining was performed using an ApoAlert DNA Fragmentation Assay kit (Clontech, Palo Alto, CA, USA) according to the manufacturer's instructions, with apoptotic cells exhibiting green nuclear fluorescence (26). Tumor tissue sections were counterstained with PI for 5-10 min to stain cell nuclei. The percentage of apoptotic cells was assessed in 10 randomly selected fields at a magnification of $x 40$. The apoptotic index was calculated as the number of apoptotic cells/total number of nucleated cells x $100 \%$.

Statistical analysis. The results obtained from cell growth experiments are expressed as the mean \pm SD. The results from different treatments were compared using two-tailed Student's $\mathrm{t}$-tests and were considered significant at a threshold of $\mathrm{p}<0.05$.

\section{Results}

Ectopic overexpression of FOXK1 increases the expression of multiple oncogenes in vitro. We developed in vitro models to examine the mechanistic role of FOXK1 in CRC biology, and we established stable transfectants with FOXK1 sense and vector (pcDNA3.1) plasmids. The overexpression of FOXK1 was confirmed by western blot analysis (Fig. 1A).

Next, we screened for potential FOXK1 target genes by ectopically expressing FOXK1 in SW480 cells and examining the subsequent changes in the expression levels of 10 major oncogenes that are reportedly involved in proliferation, transformation or apoptosis inhibition (27). The mRNA expression levels of ZEB1, ID1, Sp1, cyclin D1, $\beta$-catenin, Myc, TWIST, TERT, survivin and FOXK1 were upregulated in the stable transfectants of FOXK1 (Fig. 1B).

Our results suggest that FOXK1 activates the expression of multiple oncogenes in CRC.

FOXK1 promotes cell growth by stimulating cell proliferation. To analyze the effect of FOXK1 on cell growth, we assessed the proliferation of the stable transfectants cultured in complete cell culture medium at various time points. The cell growth percentages relative to those of transfectants carrying an empty vector were calculated. We showed that the growth rates of the SW480-FOXK1 transfectants were 103.1 \pm 1.5 , $169.6 \pm 3.1$ and $214.3 \pm 4.0 \%$ after culture for 24,48 and $72 \mathrm{~h}$, respectively (Fig. 1C). Significant differences between the FOXK1- and empty vector-transfected cells were found at 48 and $72 \mathrm{~h}(\mathrm{p}<0.05)$.

Anchorage-independent growth is one pivotal characteristic of malignant transformation. To determine whether forced expression of FOXK1 may alter the oncogenicity of CRC cells, we examined the anchorage-independent growth of the stable transfectants with the empty vector or with FOXK1 in soft agar after 14 days. SW480 and empty vector-transfected cells formed colonies in soft agar as expected, while forced expression of FOXK1 markedly enhanced colony formation in soft agar (Fig. 1D). FOXK1 expression increased the clonogenicity of the SW480 cells by $155 \%$ (Fig. 1E). Conversely, we also tested the effect of RNAi-mediated FOXK1 knockdown in the 


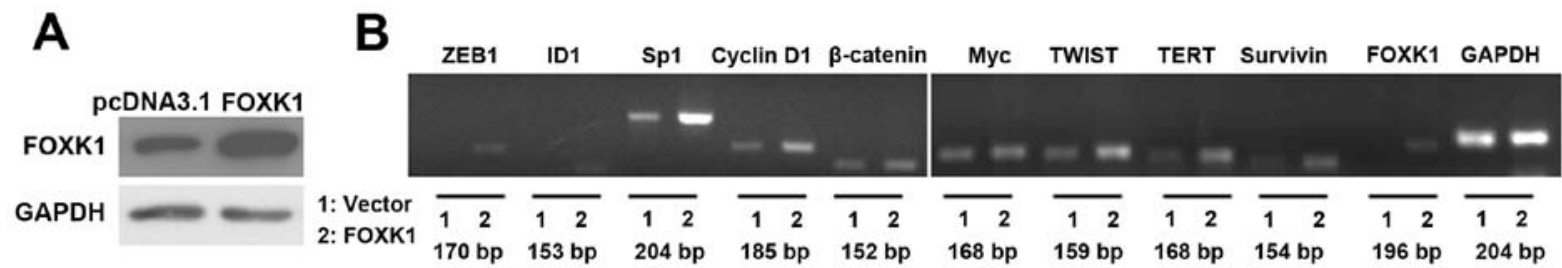

C

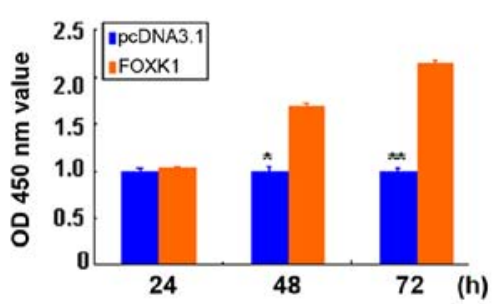

E

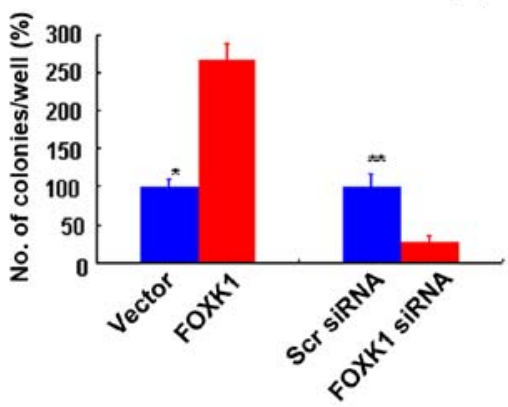

D

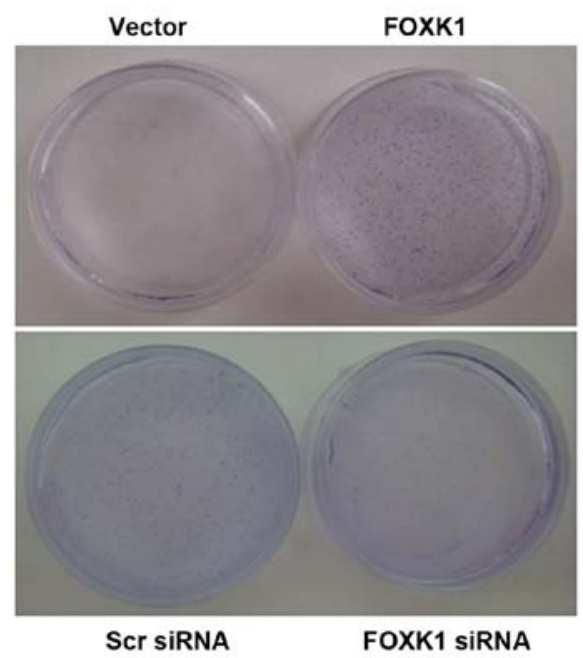

Figure 1. FOXK1 regulates multiple oncogenes and promotes the proliferation and anchorage-independent growth of CRC cells. (A) FOXK1 expression in stable transfectants of SW480 cells harboring the empty vector or expressing FOXK1, as detected by western blotting. GAPDH was used as the internal control. (B) The mRNA expression levels of ZEB1, ID1, Sp1, cyclin D1, $\beta$-catenin, Myc, TWIST, TERT, survivin and FOXK1 were upregulated in stable transfectants of FOXK1. (C) Significant differences between FOXK1- and empty vector-transfected cells were found at 48 and $72 \mathrm{~h}$ (p<0.05). (D) SW480 and empty vector-transfected cells formed colonies in soft agar as expected, while forced expression of FOXK1 markedly enhanced colony formation in soft agar. (E) FOXK1 expression increased the clonogenicity of SW480 cells by $155 \%$.

SW480 cells. FOXK1-siRNA knockdown caused a significant loss of colony-forming capacity. The clonogenicity of the suppressed SW480 cells was reduced by $71.4 \%$ (Fig. 1E).

Therefore, the overexpression of FOXK1 markedly promoted the proliferation of CRC cells.

Knockdown of FOXK1 induces G0/G1 cell cycle arrest. To assess in more detail the effect of constitutive FOXK1 expression on the growth characteristics of CRC cells in vitro, we evaluated the cell cycle distribution of the cells by flow cytometry. FOXK1 knockdown increased the proportion of cells in the G0/G1 phase and concomitantly decreased the proportion of cells in the G2/M phase of the cell cycle (Fig. 2A).

Furthermore, cell cycle-related protein expression was assessed by western blotting. Consistent with the accumulation of cells in G0/G1 phase, cyclin D1, CDK4 and CDK6 were significantly decreased in the FOXK1-siRNA-treated SW480 and SW1116 cells (Fig. 2B). The cyclin-dependent kinases (CDKs) CDK4 and CDK6, along with cyclin D1 proteins, are involved in the progression of cells through G1 phase and their entry into $\mathrm{S}$ phase. Collectively, these findings indicate that reduced expression of FOXK1 slowed cell cycle progression through the G1 phase.

Knockdown of FOXK1 induces CRC cell apoptosis. To investigate the mechanism by which FOXK1-siRNA induces growth suppression, we performed an apoptosis assay.
Apoptosis was detected by FACS analysis after double staining with PI and Annexin V-FITC. As shown in Fig. 3A, FOXK1-siRNA induced more apoptosis than scr-siRNA in the SW480 cells, indicating that FOXK1-siRNA inhibited cell growth by inducing apoptosis. Apoptotic induction was further confirmed by Hoechst 33258 staining at the single-cell level (Fig. 3B and C). Moreover, FOXK1-siRNA activated caspases 3,8 and 9, as evidenced by the increased protein level of cleaved caspases compared with that in the scr-siRNAtreated cells (Fig. 3D). Taken together, these results support the idea that knockdown of FOXK1 promotes the apoptosis of CRC cells.

Silencing of FOXK1 suppresses the growth of xenograft tumors in nude mice. To explore the effects of FOXK1 knockdown in vivo, xenograft tumors were generated by injecting SW480 cells that were stably infected with either scr-shRNA or FOXK1-shRNA. The cells were subcutaneously injected into the flanks of nude mice (Fig. 4A). As shown in Fig. 4B, compared with the scr-shRNA group, the tumors derived from FOXK1-shRNA-infected cells grew much more slowly throughout the experiment, suggesting that knockdown of FOXK1 markedly impaired the tumorigenic growth of the SW480 cells.

We next examined the protein expression of cell proliferation (Ki-67) and angiogenesis markers (CD105) in xenograft tumors. Representative immunohistochemical images of the 
A

A

Scr SiRNA
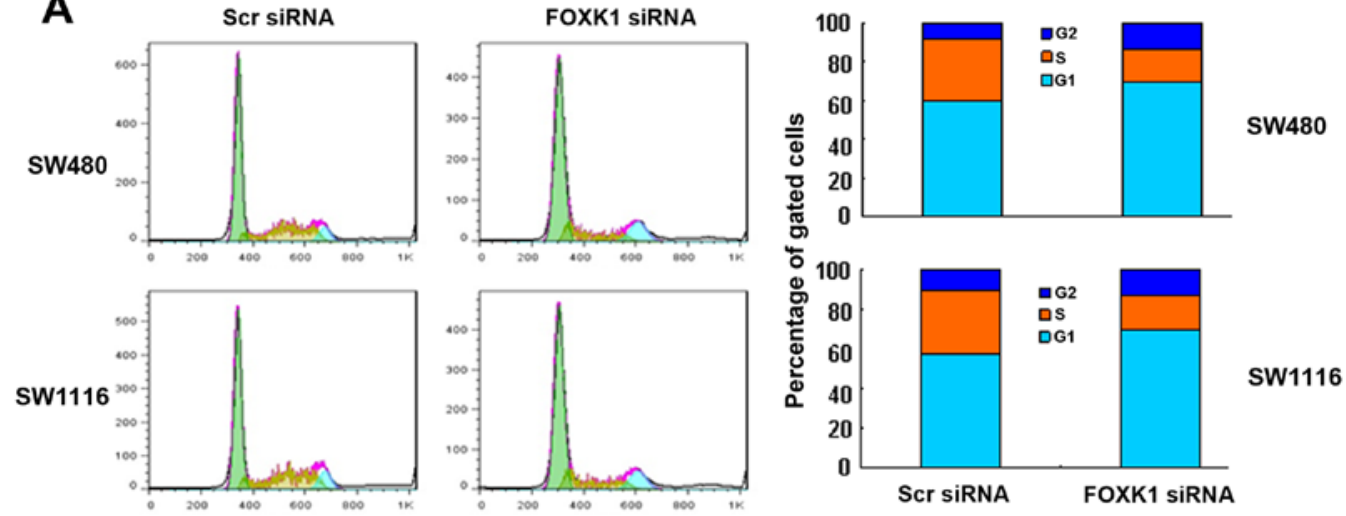

B

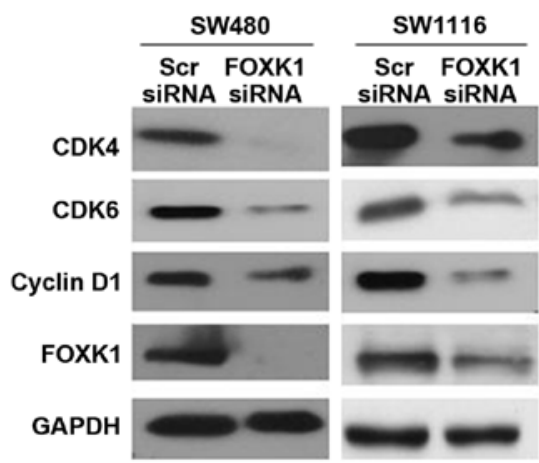

Figure 2. FOXK1 modulates cell cycle progression. (A) The effects of FOXK1-siRNA and scr-siRNA on FOXK1 expression and on the cell cycle distribution of SW480 cells were determined by flow cytometry after staining with PI. (B) Whole-cell lysates of parental SW480 cells were prepared, and protein expression was detected by western blotting. GAPDH was used as the internal control.

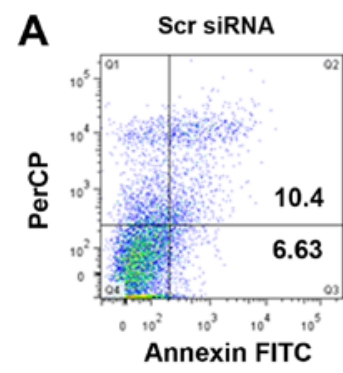

B

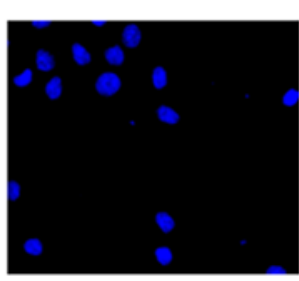

Scr SiRNA

C

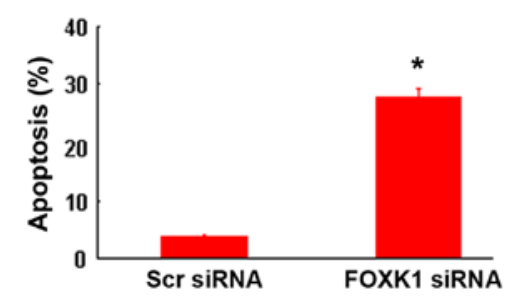

FOXK1 SIRNA
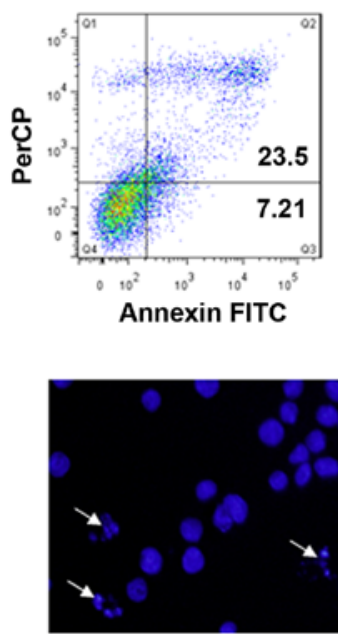

FOXK1 SIRNA

D

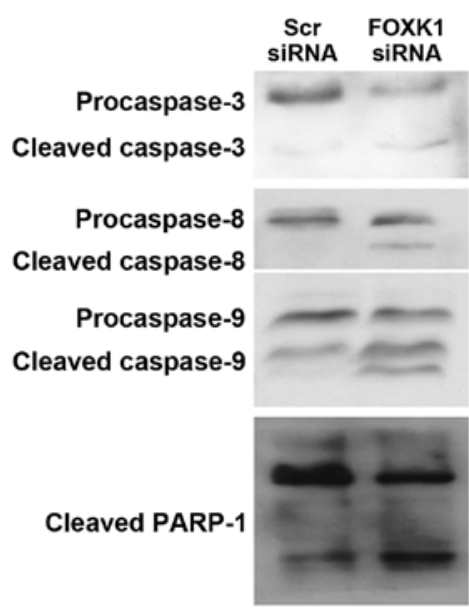

GAPDH

Figure 3. Knockdown of FOXK1 induces CRC cell apoptosis. (A) Cells transfected with scr-siRNA or FOXK1-siRNA were double-stained with Annexin V-FITC and PI, and then subjected to flow cytometric analysis. (B) SW480 cells were treated with scr-siRNA or FOXK1-siRNA, the nuclei were stained with Hoechst 33258, and the cells were visualized under a fluorescence microscope (arrows denote cells exhibiting nuclear fragmentation and condensed chromatin). (C) Histogram of the percentages of apoptotic cells after staining with Hoechst 33258 . The values are expressed as the mean \pm SEM of 3 separate experiments; ${ }^{*}$ p<0.01. (D) SW480 cells were transfected with scr-siRNA or FOXK1-siRNA, and cell lysates were prepared after 48 h. The expression levels of pro- and cleaved caspase 3,8 and 9, and PARP-1 were detected by western blotting with GAPDH as the internal control. 
A
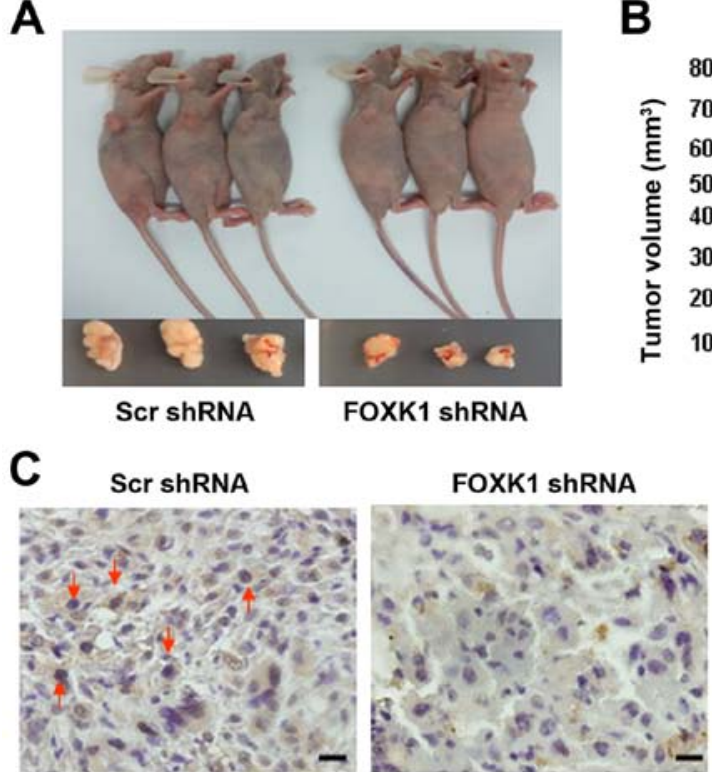

Ki-67

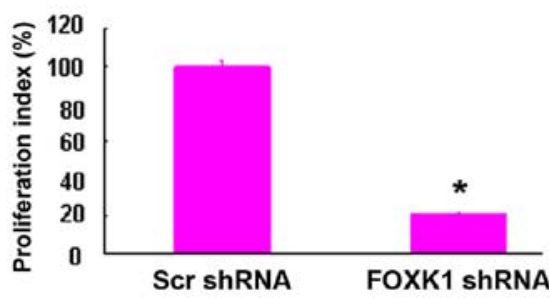

B
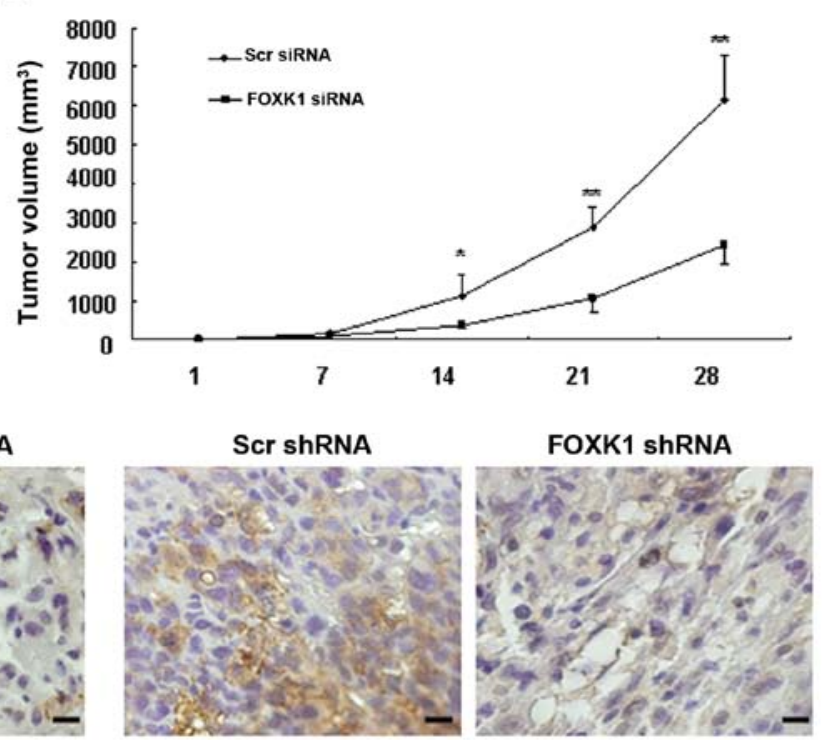

CD105

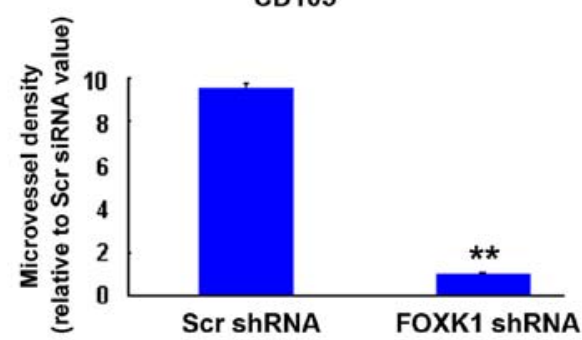

Figure 4. FOXK1 promotes CRC cell proliferation in vivo. (A) SW480 cells transfected with scr-shRNA or FOXK1-shRNA were subcutaneously injected into the right flanks of nude mice, which were sacrificed after 28 days. (B) The tumor size was measured weekly after tumor cell inoculation in each group; ${ }^{*} \mathrm{p}<0.01$ and $^{* *} \mathrm{p}<0.001$, scr-shRNA vs. FOXK1-shRNA. (C) FOXK1 knockdown significantly inhibited the proliferation of SW480 cells (Ki-67, ${ }^{*} \mathrm{p}<0.01$, vector vs. FOXK1-shRNA), and a considerable decrease in the tumor vessel density (CD105, ${ }^{* * *} \mathrm{p}<0.001$, vector vs. FOXK1-shRNA) was observed by immunohistochemistry. Scale bars, $100 \mu \mathrm{m}$.

tumors are shown in Fig. 4C. The knockdown of FOXK1 in the FOXK1 group showed significantly inhibited proliferation rates and lower tumor vessel density relative to the scr-shRNA group. These findings suggest that FOXK1-shRNA may have an inhibitory effect on tumorigenesis in vivo.

Knockdown by FOXK1-shRNA increases cell susceptibility to apoptotic stimuli in vitro and in vivo. To evaluate the role of FOXK1-siRNA-mediated knockdown in the susceptibility of cells to chemotherapy-induced apoptosis, SW480 cells were treated with or without $5-\mathrm{FU}(50 \mu \mathrm{g} / \mathrm{ml}$, using NS as the vehicle) for $48 \mathrm{~h}$ in vitro. Double staining with Annexin V and PI was then performed, followed by flow cytometric analysis to determine the apoptosis rate. As shown in Fig. 5A, the apoptotic indices of the cells transfected with FOXK1siRNA + 5-FU and scr-siRNA + 5-FU were significantly increased relative to the scr-siRNA controls.

Similarly, by Hoechst 33258 staining, the proportion of condensed nuclei-positive cells was higher in the SW480 cells transfected with FOXK1-siRNA + 5-FU ( $<<0.001$ compared with FOXK1-siRNA + 5-FU) and scr-siRNA + 5-FU (p<0.01 compared with scr-siRNA + 5-FU) than in SW480 cells transfected with the scr-siRNA (Fig. 5B).

Moreover, the antitumor effect of FOXK1-shRNA and/ or 5-FU in vivo was demonstrated by a xenograft model in nude mice (Fig. 5C). We subcutaneously injected SW480 cells that were stably infected with either scr-shRNA or FOXK1shRNA into the right flanks of nude mice, respectively. When tumor nodules became visible ( $3-5 \mathrm{~mm}$ in diameter), 5-FU was intraperitoneally injected as a co-treatment. The tumor sizes were then monitored weekly. As shown in Fig. 5C, the tumor volumes of the scr-shRNA-treated mice were markedly greater than those of the FOXK1-shRNA + 5-FU- and scr-shRNA + 5-FU-treated mice 4 weeks after injection of the SW480 cells.

Tumors injected with the combination of FOXK1-shRNA and/or 5-FU exhibited the greatest percentage of apoptotic cells. In contrast, tumors injected with scr-shRNA exhibited relatively few TUNEL-positive cells (Fig. 5D). These findings suggest that FOXK1-shRNA enhances the susceptibility of cancer cells to apoptotic triggers that are induced by 5-FU.

\section{Discussion}

In the present study, we found that the overexpression of FOXK1 increased the expression of multiple oncogenes. We also demonstrated that the antitumor efficacy of shRNA-mediated FOXK1 inhibition in cells and nude mouse xenograft models was due to the induction of cell cycle arrest and apoptosis. These results indicate that FOXK1-shRNA may 
A
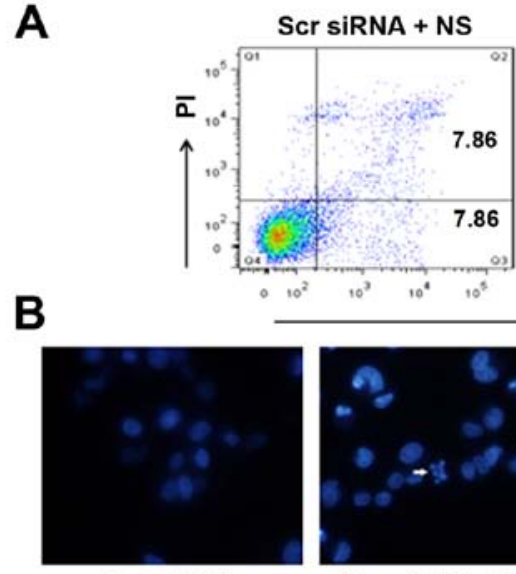

Scr siRNA

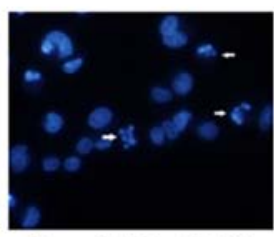

Scr siRNA + 5-FU
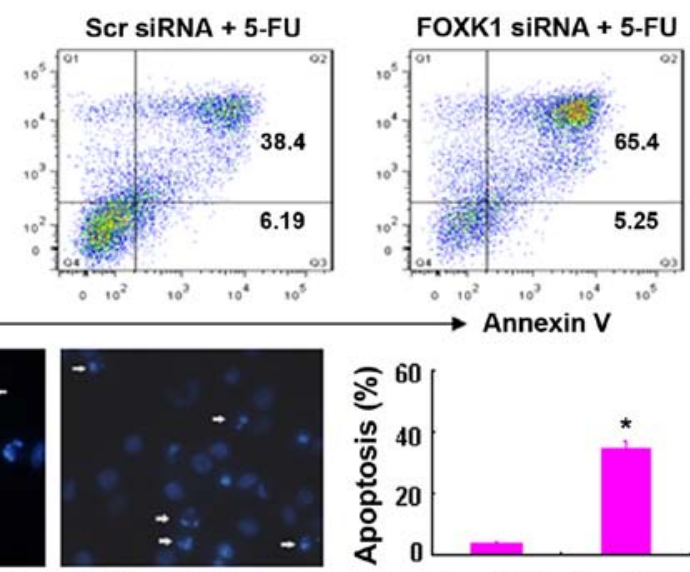

FOXK1 siRNA + 5-FU
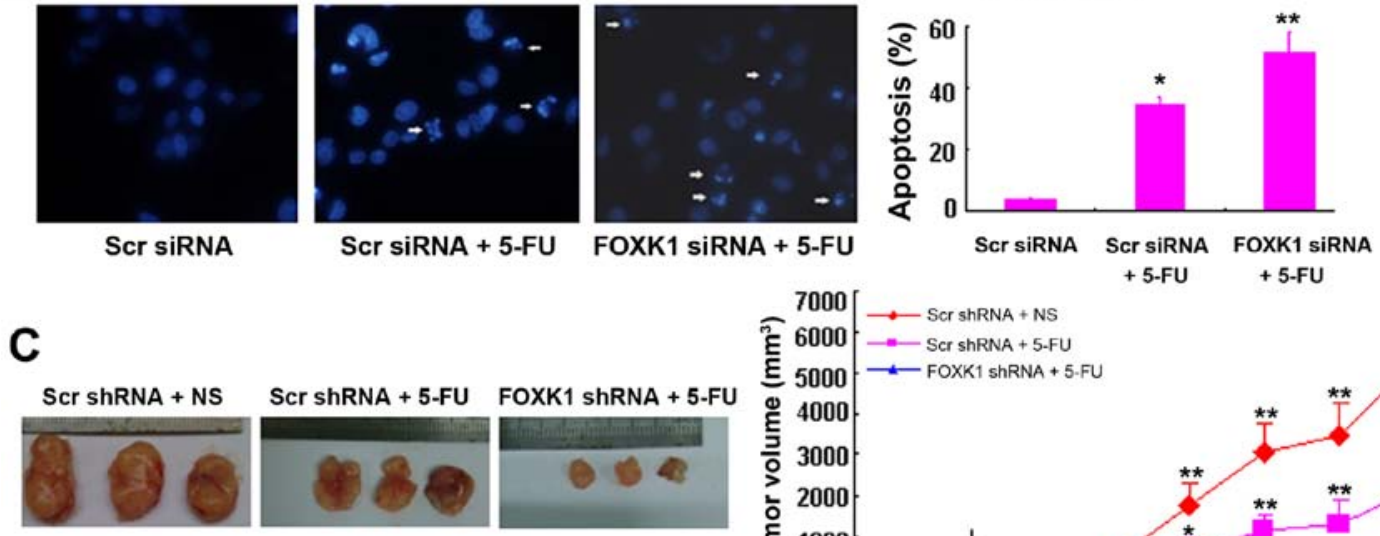

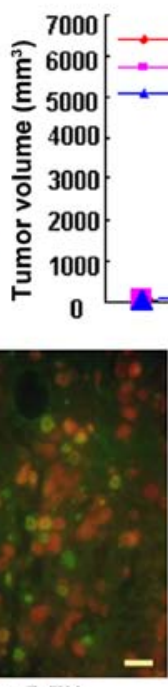

Scr shRNA + 5-FU

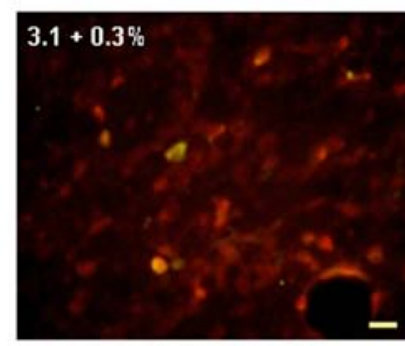

Scr shRNA + NS
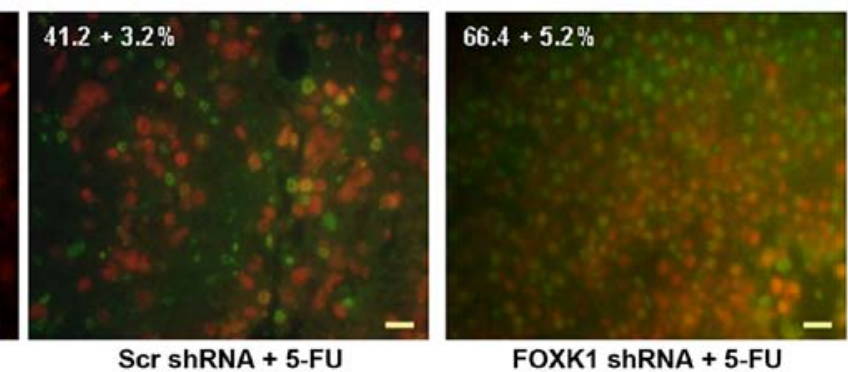

Figure 5. Knockdown of FOXK1 with shRNA sensitizes cancer cells to 5-FU-induced apoptosis in vitro and in vivo. (A) Knockdown of cells with FOXK1-siRNA increased the cell susceptibility to apoptotic stimuli. Cells transfected with scr-siRNA or FOXK1-siRNA were treated with 5-FU for 48 h, double-stained with Annexin V-FITC and PI, and subjected to flow cytometric analysis to detect apoptosis. (B) By Hoechst 33258 staining, the percentages of condensed nucleipositive cells were higher in the SW480 cells transfected with FOXK1-siRNA + 5-FU ( ${ }^{* *}$ p $<0.001$ compared with FOXK1-siRNA + 5-FU) and scr-siRNA + 5-FU ( ${ }^{*}$ p $<0.01$ compared with scr-siRNA + 5-FU) when compared with this percentage in the SW480 cells transfected with scr-siRNA. (C) Lentivirus-transduced SW480 cells were subcutaneously injected into mice. When the tumor nodules became visible (7 days later), 5 -FU (50 $\mu \mathrm{g} / \mathrm{kg}$, once $/ 2 \mathrm{days}) \mathrm{was}$ administered by intraperitoneal injection. Tumor growth was monitored in 3 dimensions and was expressed as the tumor volume in cubic millimeters. The arrow indicates the time at which intraperitoneal injections were conducted. The data are the pooled averages \pm standard errors of the means of the tumor volumes for each of the 3 animals/group; ${ }^{*}$ p $<0.01$ and ${ }^{* *}$ p $<0.001$, scr-shRNA + 5-FU vs. scr-shRNA; ${ }^{* *}$ p $<0.001$, scr-shRNA vs. FOXK1-shRNA + 5-FU. (D) Spontaneous apoptosis in tumor tissues as visualized by TUNEL staining as a marker of apoptosis (apoptotic cells are green). The tumor section was counterstained with PI for 5-10 min. The number of apoptotic cells was assessed in 3 randomly selected fields, viewed at a magnification of x40, on each slide. The apoptotic index was calculated as the number of apoptotic cells/total number of nucleated cells x $100 \%$. NS was used as the vehicle. Scale bars, $100 \mu \mathrm{m}$.

represent a novel and potent therapeutic agent, on its own or in conjunction with 5-FU, for the treatment of colon cancer.

Members of the Fox transcription factor family have been identified in several vertebrate cell lineages. Fox proteins have similar binding specificity for a core DNA sequence, $(\mathrm{T} / \mathrm{C})(\mathrm{A} / \mathrm{C}) \mathrm{AA}(\mathrm{C} / \mathrm{T}) \mathrm{A}(28,29)$, and they display conserved amino acid sequences in the putative recognition helix. There is evidence that FOX proteins have a central function in established cancers. For example, FOXA1, the most extensively studied member of the family, is upregulated in nearly all cancers, including breast (30), bladder (31), prostate (32) and pancreatic cancers (33). This protein contributes to many of the typical characteristics of cancer, including increased proliferation, resistance to cell death, and increased invasion and metastasis. Wang et al showed that FOXK1 protein levels are elevated in human CRCs (24). However, the use of FOXK1 as a target in CRC biological therapy has yet to be established. The present study showed that ectopic FOXK1 expression significantly induced the RNA levels of multiple oncogenes that are involved in neoplastic transformation, apoptosis, the cell cycle and proliferation, including ZEB1, ID1, Sp1, cyclin D1, $\beta$-catenin, Myc, TWIST, c-Jun and survivin. Among these targets, Myc and cyclin D1 are involved in cell cycle regulation. Survivin and $\beta$-catenin are involved in neoplastic transformation. The transcription factors TWIST, AP-1, Sp1, ZEB1 and ID1, as well as epithelial-to-mesenchymal transition inducers, underlie the phenotypic conversion from epithelial cells to mesenchymal cells. Thus, FOXK1 acts as an oncogene in CRC.

The oncogenic function of FOXK1 in colon cancer was further investigated using in vitro and in vivo assays. 
siRNA-mediated FOXK1 knockdown had a marked growth suppression effect under both anchorage-dependent and -independent culture conditions, and it also reduced tumor size in nude mice. Flow cytometry revealed that FOXK1 knockdown caused significant G1/G0 arrest, and a concomitant reduction of CDK4/CDK6 expression was apparent. Furthermore, we found that FOXK1 knockdown increased apoptosis, a finding further confirmed by staining with Hoechst 33258. Notably, apoptotic induction by FOXK1-siRNA was mediated through the intrinsic and extrinsic caspase-dependent pathways. Taken together, these results indicate that FOXK1 is involved in cell growth and apoptosis in CRC.

The drug 5-FU is an important component of standard chemotherapy protocols for various solid tumors, including CRCs $(34,35)$. In the present study, we further investigated the in vitro and in vivo effects of combination treatment with FOXK1-shRNA and 5-FU on colon cancer growth. According to flow cytometric analysis and Hoechst 33258 staining, knockdown of FOXK1 resulted in a greater proportion of 5-FU-induced apoptotic cells. We also found that the volumes of xenograft tumors were significantly reduced when treated with a combination of FOXK1-shRNA and 5-FU.Furthermore, TUNEL staining demonstrated that FOXK1-shRNA could further enhance 5-FU-induced apoptosis. To the best of our knowledge, no studies have yet shown the therapeutic value of RNAi-mediated FOXK1 knockdown in conjunction with 5-FU in CRC.

The present study demonstrates that FOXK1 acts as an oncogene in CRC. FOXK1 knockdown inhibits cell growth and promotes apoptosis in CRC cells in vitro. Furthermore, FOXK1-shRNA hinders tumor progression and results in outright regression when combined with 5-FU in vivo. Thus, our current data provide direct evidence that a combination of RNAi-mediated FOXK1 knockdown and 5-FU may be a potent strategy for colon cancer therapy.

\section{References}

1. Finlay IG and McArdle CS: Effect of occult hepatic metastases on survival after curative resection for colorectal carcinoma. Gastroenterology 85: 596-599, 1983.

2. Kemp Z, Thirlwell C, Sieber O, Silver A and Tomlinson I: An update on the genetics of colorectal cancer. Hum Mol Genet 13 (Suppl 2): R177-R185, 2004.

3. Konishi M, Kikuchi-Yanoshita R, Tanaka K, Muraoka M, Onda A, Okumura Y, Kishi N, Iwama T, Mori T, Koike M, et al: Molecular nature of colon tumors in hereditary nonpolyposis colon cancer, familial polyposis, and sporadic colon cancer. Gastroenterology 111: 307-317, 1996.

4. van Erning FN, Crolla RM, Rutten HJ, Beerepoot LV, van Krieken JH and Lemmens VE: No change in lymph node positivity rate despite increased lymph node yield and improved survival in colon cancer. Eur J Cancer 50: 3221-3229, 2014.

5. Mazet F, Yu JK, Liberles DA, Holland LZ and Shimeld SM: Phylogenetic relationships of the Fox (Forkhead) gene family in the Bilateria. Gene 316: 79-89, 2003.

6. Bieller A, Pasche B, Frank S, Gläser B, Kunz J, Witt K and Zoll B: Isolation and characterization of the human forkhead gene FOXQ1. DNA Cell Biol 20: 555-561, 2001.

7. Ackermann S, Kocak H, Hero B, Ehemann V, Kahlert Y, Oberthuer A, Roels F, Theißen J, Odenthal M, Berthold F, et al: FOXP1 inhibits cell growth and attenuates tumorigenicity of neuroblastoma. BMC Cancer 14: 840, 2014

8. Stoll SW, Stuart PE, Swindell WR, Tsoi LC, Li B, Gandarillas A Lambert S, Johnston A, Nair RP and Elder JT: The EGF receptor ligand amphiregulin controls cell division via FoxM1. Oncogene 35: 2075-2086, 2016.
9. Dai B, Gong A, Jing Z, Aldape KD, Kang SH, Sawaya R and Huang S: Forkhead box M1 is regulated by heat shock factor 1 and promotes glioma cells survival under heat shock stress. J Biol Chem 288: 1634-1642, 2013.

10. Kikuchi K, Hettmer S, Aslam MI, Michalek JE, Laub W, Wilky BA, Loeb DM, Rubin BP, Wagers AJ and Keller C: Cell-cycle dependent expression of a translocation-mediated fusion oncogene mediates checkpoint adaptation in rhabdomyosarcoma. PLoS Genet 10: e1004107, 2014.

11. Burgering BM: A brief introduction to FOXOlogy. Oncogene 27: 2258-2262, 2008.

12. Ouyang W, Beckett O, Flavell RA and Li MO: An essential role of the Forkhead-box transcription factor Foxol in control of T cell homeostasis and tolerance. Immunity 30: 358-371, 2009.

13. Schimmel J, Eifler K, Sigurðsson JO, Cuijpers SA, Hendriks IA, Verlaan-de Vries M, Kelstrup CD, Francavilla C, Medema RH, Olsen JV, et al: Uncovering SUMOylation dynamics during cellcycle progression reveals FoxM1 as a key mitotic SUMO target protein. Mol Cell 53: 1053-1066, 2014

14. Crose LE, Galindo KA, Kephart JG, Chen C, Fitamant J, Bardeesy N, Bentley RC, Galindo RL, Chi JT and Linardic CM: Alveolar rhabdomyosarcoma-associated $P A X 3-F O X O 1$ promotes tumorigenesis via Hippo pathway suppression. J Clin Invest 124: 285-296, 2014.

15. Nucera C, Eeckhoute J, Finn S, Carroll JS, Ligon AH, Priolo C, Fadda G, Toner M, Sheils O, Attard M, et al: FOXAl is a potential oncogene in anaplastic thyroid carcinoma. Clin Cancer Res 15: 3680-3689, 2009.

16. Xia L, Huang W, Tian D, Zhang L, Qi X, Chen Z, Shang X, Nie Y and $\mathrm{Wu}$ K: Forkhead box Q1 promotes hepatocellular carcinoma metastasis by transactivating ZEB2 and VersicanV1 expression. Hepatology 59: 958-973, 2014.

17. Wang Z, Banerjee S, Kong D, Li Y and Sarkar FH: Downregulation of Forkhead Box M1 transcription factor leads to the inhibition of invasion and angiogenesis of pancreatic cancer cells. Cancer Res 67: 8293-8300, 2007.

18. Cui YM, Jiao HL, Ye YP, Chen CM, Wang JX, Tang N, Li TT, Lin J, Qi L, Wu P, et al: FOXC2 promotes colorectal cancer metastasis by directly targeting MET. Oncogene 34: 4379-4390, 2015.

19. Li D, Mei H, Qi M, Yang D, Zhao X, Xiang X, Pu J, Huang K, Zheng L and Tong Q: FOXD3 is a novel tumor suppressor that affects growth, invasion, metastasis and angiogenesis of neuroblastoma. Oncotarget 4: 2021-2044, 2013.

20. Zhang Y, Zhang S, Wang X, Liu J, Yang L, He S, Chen L and Huang J: Prognostic significance of FOXP1 as an oncogene in hepatocellular carcinoma. J Clin Pathol 65: 528-533, 2012.

21. Ko YS, Cho SJ, Park J, Kim Y, Choi YJ, Pyo JS, Jang BG, Park JW, Kim WH and Lee BL: Loss of FOXO1 promotes gastric tumour growth and metastasis through upregulation of human epidermal growth factor receptor $2 /$ neu expression. Br J Cancer 113: 1186-1196, 2015.

22. Yan X, Zhou H, Zhang T, Xu P, Zhang S, Huang W, Yang L, $\mathrm{Gu} X, \mathrm{Ni} R$ and Zhang T: Downregulation of FOXP2 promoter human hepatocellular carcinoma cell invasion. Tumour Biol 36: 9611-9619, 2015.

23. Huang JT and Lee V: Identification and characterization of a novel human FOXK1 gene in silico. Int J Oncol 25: 751-757, 2004.

24. Wang W, Li X, Lee M, Jun S, Aziz KE, Feng L, Tran MK, Li N, McCrea PD, Park JI, et al: FOXKs promote Wnt/ $\beta$-catenin signaling by translocating DVL into the nucleus. Dev Cell 32: 707-718, 2015.

25. Wang J, Yang Y, Xia HH, Gu Q, Lin MC, Jiang B, Peng Y, Li G, An X, Zhang Y, et al: Suppression of FHL2 expression induces cell differentiation and inhibits gastric and colon carcinogenesis. Gastroenterology 132: 1066-1076, 2007.

26. Tu SP, Liston P, Cui JT, Lin MC, Jiang XH, Yang Y, Gu Q, Jiang SH, Lum CT, Kung HF, et al: Restoration of XAF1 expression induces apoptosis and inhibits tumor growth in gastric cancer. Int J Cancer 125: 688-697, 2009.

27. Cheng Y, Geng H, Cheng SH, Liang P, Bai Y, Li J, Srivastava G, $\mathrm{Ng} \mathrm{MH}$, Fukagawa T, Wu X, et al: KRAB zinc finger protein ZNF382 is a proapoptotic tumor suppressor that represses multiple oncogenes and is commonly silenced in multiple carcinomas. Cancer Res 70: 6516-6526, 2010.

28. Biggs WH III, Cavenee WK and Arden KC: Identification and characterization of members of the FKHR (FOX O) subclass of winged-helix transcription factors in the mouse. Mamm Genome 12: 416-425, 2001. 
29. Lalmansingh AS, Karmakar S, Jin Y and Nagaich AK: Multiple modes of chromatin remodeling by Forkhead box proteins. Biochim Biophys Acta 1819: 707-715, 2012.

30. Badve S, Turbin D, Thorat MA, Morimiya A, Nielsen TO, Perou CM, Dunn S, Huntsman DG and Nakshatri H: FOXA1 expression in breast cancer - correlation with luminal subtype A and survival. Clin Cancer Res 13: 4415-4421, 2007.

31. Reddy OL, Cates JM, Gellert LL, Crist HS, Yang Z, Yamashita H, Taylor JA III, Smith JA Jr, Chang SS, Cookson MS, et al: Loss of FOXA1 drives sexually dimorphic changes in urothelial differentiation and is an independent predictor of poor prognosis in bladder cancer. Am J Pathol 185: 1385-1395, 2015.

32. McMullin RP, Dobi A, Mutton LN, Orosz A, Maheshwari S, Shashikant CS and Bieberich CJ: A FOXA1-binding enhancer regulates Hoxb13 expression in the prostate gland. Proc Natl Acad Sci USA 107: 98-103, 2010.
33. Song Y, Washington MK and Crawford HC: Loss of FOXA1/2 is essential for the epithelial-to-mesenchymal transition in pancreatic cancer. Cancer Res 70: 2115-2125, 2010.

34. Wu Y, Guo Z, Zhang D, Zhang W, Yan Q, Shi X, Zhang M, Zhao Y,Zhang Y, Jiang B, et al: A novel colon cancer gene therapy using rAAV-mediated expression of human shRNA-FHL2. Int J Oncol 43: 1618-1626, 2013.

35. Li X, Kong X, Kong X, Wang Y, Yan S and Yang Q: 53BP1 sensitizes breast cancer cells to 5-fluorouracil. PLoS One 8: e74928, 2013. 